\title{
Caries removal using lasers
}

\author{
Abstracted from \\ Montedori A, Abraha I, Orso M, D'Errico PG, Pagano S, Lombardo G. \\ Lasers for caries removal in deciduous and permanent teeth. Cochrane Database Syst Rev 2016; 9: Art. No.:CD010229. DOI: 10.1002/14651858. \\ CD010229.pub2 \\ Address for correspondence: Luisa Fernandez Mauleffinch, Managing Editor, Cochrane Oral Health Group, School of Dentistry, The University of \\ Manchester, JR Moore Building, Oxford Road, Manchester, M13 9PL, UK. E-mail: luisa.fernandez@manchester.ac.uk
}

\section{Question: Does a laser remove caries as effectively as mechanical drilling?}

Data sources Cochrane Oral Health Trials Register, Cochrane Central Register of Controlled Trials (CENTRAL), Medline Ovid, Embase Ovid, ProQuest Dissertations and Theses, Zetoc, ISI Web of Knowledge, US National Institutes of Health Ongoing Trials Register ClinicalTrials.gov, and the WHO International Clinical Trials Registry Platform for ongoing trials up to June 2016.

Study selection Randomised controlled trials, split-mouth trials and cluster-randomised trials comparing laser ablation to drill removal of caries with no restriction in language or participants' age.

Data extraction and synthesis Studies were selected and reviewed independently by two reviewers and standard data items extracted. The reviewers assessed the risk of bias of all studies using Cochrane's 'Risk of bias ' tool. For all dichotomous outcomes, risk ratios (RR) with $95 \%$ of confidence intervals $(\mathrm{Cl})$ were calculated, and mean difference (MD) was calculated for continuous data. Primary outcomes were effectiveness in caries excavation and reports of pain. Data were combined using random-effects models. Main results for caries removal were summarised and presented using GRADE proGDT software. Meta-analyses were performed on studies that reported quantitative data.

Results Six split-mouth randomised controlled trials and three parallelgroup randomised trials involving 1,498 primary and permanent teeth from 662 participants ranging from 3.5 to 84 years old were included. Seven hundred and seventy-seven teeth were treated with laser only, 732 with mechanical drills only, and 12 teeth were treated with both techniques in the same tooth on separate caries.

Only four studies evaluated caries removal. Of those four, only two reported quantitative data. After meta-analysis, the results showed no significant difference in effectiveness of caries removal between the two treatment methods ((RR) $1.00,95 \%(\mathrm{Cl}) 0.99$ to 1.01$)$. Of the five studies that assessed pain, three studies using five-point or six-point pain scale reported less pain experienced using laser. Two studies did not provide complete data for analysis. As for the secondary outcomes of marginal integrity of restorations (three studies), durability (four studies), recurrent caries (two studies), pulpal inflammation or necrosis (four studies), overall results showed no evidence of a difference.

This paper is based on a Cochrane Review published in the Cochrane Library 2016, issue 9 (see www.thecochranelibrary.com for information). Cochrane Reviews are regularly updated as new evidence emerges and in response to feedback, and the Cochrane Library should be consulted for the most recent version of the review.
When considering the need for anaesthesia (four studies), and participant discomfort (five studies), the overall results showed that the need for anaesthesia and participant discomfort was lower with the laser treatment. Interestingly, the one study that reported operator preference showed that dentists preferred conventional preparation method over laser $(P<0.001)$.

Conclusions There was insufficient evidence to show that laser removal of caries was more or less efficient than traditional mechanical technique. However, there was some low quality evidence in favour of laser therapy for pain control, need for anaesthesia and patient comfort.

\section{Commentary}

A wide range of database was searched with no limitation to language or age. The included studies came from USA, UK, Germany, Taiwan, China, Bulgaria and Turkey. All studies were randomised trials. The follow-up period ranged from one week to 24 months.

While the analysis demonstrated the same effectiveness in caries removal by both laser and drill methods (based on two studies), and that patients reported less incidence of pain and higher acceptance by the laser method, the quality of the evidence was low. Review authors concluded that there was insufficient evidence to support the use of laser due to the low quality of the evidence. Overall, trials had small sample sizes and the majority were at unclear or high risk of selection, performance, detection, attrition and reporting bias. Five studies received funding from device manufacturers which further increased the other bias.

A previous systematic review that investigated laser technology for caries removal in the medical literature (Jacobsen 2011) reached a similar conclusion.

Prevalence of dental caries remains very high worldwide. It is the main cause of tooth loss. Traditional mechanical drilling techniques with the accompanying noise, vibration and discomfort, can trigger anxiety and dental phobia in patients, especially for children, and discourage them from seeking dental care. Alternative excavation tools/techniques like laser that are more conservative and less traumatic may induce less dental discomfort, anxiety and fear. However, due to the higher cost of laser, and the uncertainty about laser's ability to remove old restorations and secondary caries, the use of laser is still limited. Higher quality randomised clinical trials with longer follow-up periods and larger sample sizes are warranted. 